\title{
Automated single view 3D Texture Mapping and Defect Localisation of Thermography Measurements on large Components utilising an industrial robot and a laser system
}

\author{
Somen Dutta ${ }^{1}$, Prof. Dr.-Ing. Klaus Drechsler ${ }^{2}$, Prof. Dr.-Ing. Michael Kupke ${ }^{3}$, Dr. Alfons Schuster ${ }^{4}$, \\ Jens-Peter Tuppatsch ${ }^{5}$
}

\author{
1 German Aerospace Centre, Germany, somen.dutta@dlr.de \\ 2 Technical University of Munich, Germany, drechsler@lcc.mw.tum.de \\ 3 German Aerospace Centre, Germany, michael.kupke@dlr.de \\ 4 German Aerospace Centre, Germany, alfons.schuster@dlr.de \\ 5 German Aerospace Centre, Germany, jens.tuppatsch@dlr.de
}

\begin{abstract}
Autonomous measurement with NDT system like thermography on a large aircraft component (e.g. fuselage sections) is a major challenge for standard industry robot. Many factors may affect the absolute positioning accuracy of created robot TCP positions. $0.1^{\circ}$ absolute robot inaccuracy (TCP) can cause for 1 meter working distance up to $3 \mathrm{~mm}$ position error. Another major drawback of thermography system is that certified examiners evaluate every 2D thermography image per measuring field one by one. This unhandy and less intuitive method for large number of numerical data is inappropriate for industrial applications. There are plenty of existing methods and technologies like Stereo vision method, using 3D feature or multi-view method for 3D texture mapping. These methods require either a second camera or time consuming additional processes or several images from every single measuring field.

This paper introduces development of a prototype robot based thermography measuring system and a new method for 3D evaluation along with visualization of thermography results, which minimizes drawbacks described above. Evaluation in 3D components coordinate, gives complex but complete 3D defect propagation knowledge. DLR-AQP department is working on two different methods to calculate 2D-3D camera projection parameters. Method one projects predefined 3D points per measuring field by using laser projector and applies 2D-3D point correspondence algorithm. The second Method uses robot position and applies kinematic-coordinate-relationships algorithm. For both methods single thermal image is captured by using thermography camera, which is also used for NDT. The first method is applicable in laboratories as well as in production with and without any manipulator. This method enables also contactless localization of detected defects. The second method requires robot. About accuracy, reproducibility and flexibility of both methods have been compared and discussed in this paper. The 3D results are to be imported directly into the CAD environment for further structure analysis.
\end{abstract}

\section{Introduction}

During the manufacturing process, it is desirable to examine parts to confirm they are defect free and fit for service without destroying the part. Large object measurements are always difficult and very expensive to automate. The main reason is that most large objects are bigger than the working envelope of measuring machines. Furthermore, modulated thermal waves during Lock-In Thermography diffuses laterally to the surrounding surfaces of measurement filed, which prevents continuous measuring the very next measurement field. Therefore, for large components, the thermography measurement position has to be distributed systematically to get homogenous intensity of measurement field. Another major drawback of a thermography system is that, certified examiners have to evaluate every 2D thermography image per measuring field one by one. This unhandy and less intuitive method for large number of numerical data is inappropriate for industrial applications. These 2D images are projections of measurement objects, which prevent to demine the accurate defect dimension and 3D defect propagation [1]. Evaluation in 3D components coordinate, gives complex but complete 3D defect propagation knowledge.

To reduce production cost, industry has a growing interest in the manufacturing process (see figure 1) automation with robotic systems. However, it is known that robots have better repeatability than absolute positioning accuracy. Normally robot repeatability accuracy is about 0.1 to $0.2 \mathrm{~mm}$ but the absolute positioning inaccuracy may be up to $20 \mathrm{~mm}$ or even more if not properly calibrated. The major robotic problem can be basically divided into positioning and path planning. Robot Tool-Centre Point (TCP) positional accuracy depends mostly on robot pose and their orientation. Many other factors like gravitational or deflecting forces may affect the absolute positional accuracy of robot TCP positions. For example, $0.1^{\circ}$ absolute robot inaccuracy (TCP) can cause for 1 meter working distance up to $3 \mathrm{~mm}$ positional error. Many fields of investigation exist to increase the accuracy of industrial robots like: robot calibration, process development and control system etc. Robot calibration improves the accuracy of positioning by reducing the deviation between the 
commanded pose and the real one. Different techniques also exist to compensate these deviations. The sensor based method offers good position accuracy. However, it's implementation on an existing robot is difficult. Many problems could be encountered like the positioning of sensors on the robot structure and their integration in the control loop besides the extra cost due to the integration of these sensors.

In order to overcome this limitation and retrieve a 3D thermography texture for the observed object, DLR Augsburg has developed both hardware and software components. The approach at DLR Augsburg of a robot based measuring system for industrial thermography applications and concept of 3D-Thermography visualisation procedure with a thermography end-effector has been presented in previous papers [2, 3 and 4]. The essential information required to generate 3D thermography are object's geometry, location and orientation of objects with reference to Camera, particularly across separate images from all measurement field [5]. There are plenty of existing methods and technologies for 3D texture mapping like the stereo vision method, using 3D features, or the multi-view method. These methods require either a second camera [5] or time consuming additional processes like several images taken from different views for each single measuring field. To address limitations of existing 3D thermography texture mapping solutions, DLR Augsburg proposes a new 3D visualisation (see Figure 1) method using thermography camera, which is also used for NDT, and a laser system mounted above the robotic cell, eliminates the uncertainty of robot's position accuracy. With an easy calibration process within a few minutes the laser system is calibrated to the component coordinate system and projects predefined 3D points for every measuring field. These 3D points are captured by the thermography camera; afterwards a 2D-3D point correspondence algorithm is applied to calculate 2D-3D camera projection parameters. The second Method (Figure 2) uses the robot pose and thereby a kinematic-coordinaterelationships algorithm is applied to the images. For both methods single thermal image is captured using a thermography camera. The first method is suited for research and development as well as for production inspection with or without using a robot for camera positioning while the second method requires a robot. The first method enables also contactless localisation of detected defects. Accuracy, reproducibility and flexibility of both methods are compared and discussed in this paper. The 3D results from above mentioned methods are imported directly into the CAD environment for further structural analysis.

The rest of this paper is organized as follows: The feasibility of new concept for 3D texture mapping has been analysed in Section 2. In Section 3 has been discusses about the results of geometric camera calibration and hand-eye-camera calibration. This calibration is required for 3D visualisation. Section 4 provides 3D visualisation results from captured data of both methods, mentioned above. Section 5 will give an overview how to handle generated 3D data for further structural analysis as well as represents layer wise (according to chosen measurement frequency) 3DThermotomography model. This 3D-Thermotomography visualisation enables details $3 \mathrm{D}$ evaluation. The paper is concluded in Section 6.

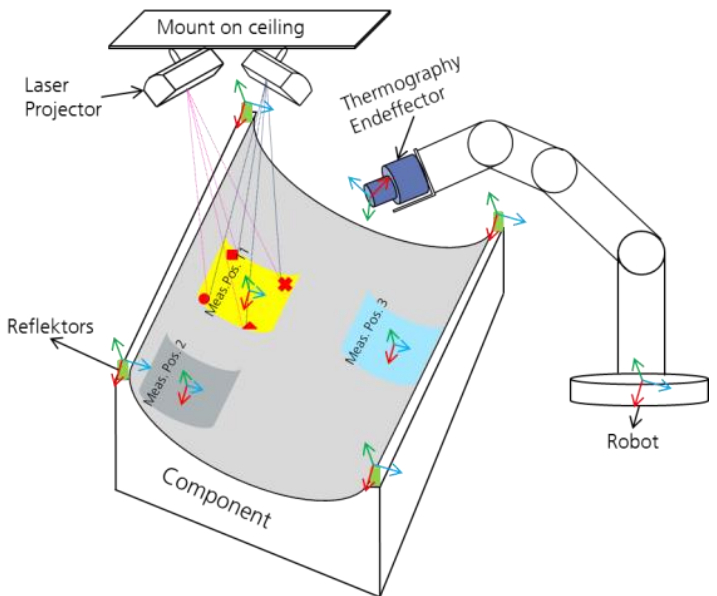

Figure 1:2D-3D point correspondence approach

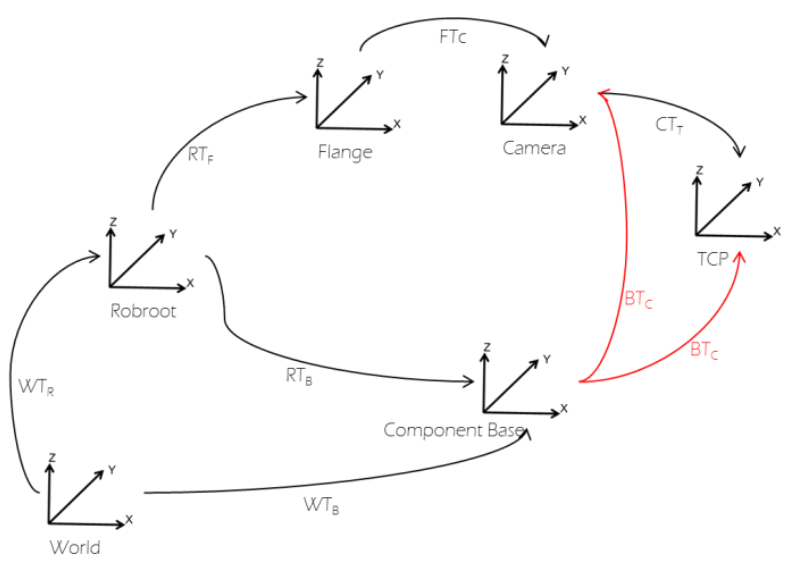

Figure 2: Kinematic coordinate chain approach

\section{Feasibility Study of new concept}

Since last 20 years, there is a keen research interest in automated feature recognition from image for 3D reconstruction. This recognition may be in terms of vanishing points or lines [7, 8], co-planarity [9], spatial inter-relationship of features [10] and camera constraints [11]. The most critical point in this process depends on precise and reliable identification of belonging structural elements, which is provided interactively by the user. Other approach to obtain scene knowledge is unsupervised image-based modelling [12]. For the new concept (see above), the provided respectively projected laser points, which are visible by thermography camera due to local surface temperature difference, have to be recognised automatically. For 3D reconstruction minimum 4 points are required. Previous results have shown that for a distance more than $500 \mathrm{~mm}$ between laser projector and component, only one laser spot can be detected at once by thermography camera. Due to safety reason laser projector has less performance and is not able to generate enough temperature difference for more than one point. Hence, for all experiments, 9 points are projected one by one for every 
single measuring field. This drawback can easily be solved either by using high performance laser or by using more laser projector at once or by using more sensible camera. DLR Augsburg has developed cross correlation based software to detect the laser points automatically from thermal image (Figure 3a)Therefore, a similar template like laser spot in $32 \mathrm{x}$ 32 Pixel size has been generated (Figure $3 b$ ). As well as, several filters has been used to minimise noise from thermal image. Although the software is very stable for detection, there are many possible factors which may influence the detection. For example, point size, laser projector distance, projection time, surface reflection and materials temperature absorption degree. Experimental evaluation has been performed to find out the parameters from above influential effects.

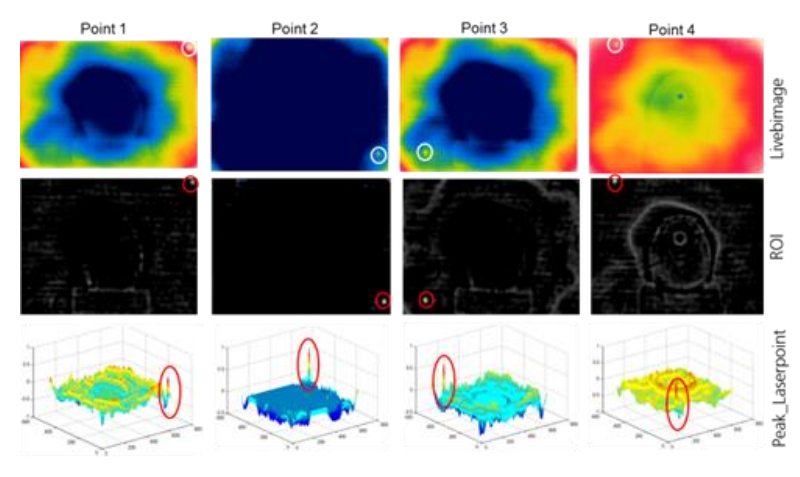

(a) Laser point detection (b) Correlation template

Figure 3: (a) Laser point detection, (b) Correlation template

Right at the beginning of the test series, it was noticed that changing the projector's distance has no influence on the laser point detection. The minimal (e.g. $2 \mathrm{~m}$ ) change in distance of the projector (according to intensity definition) leads to no measurable change in the point recognition. Therefore, the distance was kept constant during the experiment.

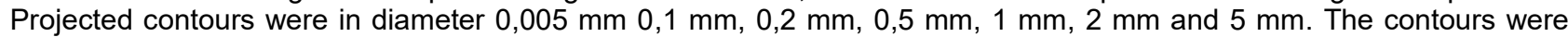
taken with the thermographic camera at a distance of $250 \mathrm{~mm}$ and $500 \mathrm{~mm}$. This process was performed twice (once with one laser projector and a second time with two projectors). The contours were projected on the $5 \mathrm{~mm}$ and $2 \mathrm{~mm}$ thick plates respectively on the tool side (more reflection) and on the non-tool side (less reflection). The plates were made of different CFRP materials. Further results show above mentioned parameter variations have no influence upon plates which have $3 \mathrm{~mm}$ thickness difference.

Depending on the fibre orientation and position (the tool side reflects more than the opposite side or the non-tool side) of the plate, the laser beam reflects differently (angle of incidence equal to the angle of reflection) and is registered accordingly in the thermographic camera. Since the camera distance to the component was too small (in this case 250 $\mathrm{mm}$ ), the thermographic camera could not recognise the larger contours as a point due to random reflection properties. Thus, the evaluation algorithm for laser point detection within a plate has found different pixel values in image coordinates. The deviation was more than 2 Pixel. Similar or more deviation effects have been also observed for this configuration by using two projectors, as the projection was not congruent due to not optimised calibration. The deviation in this case was between 1 and 2 Pixel. The evaluation of this result is that the camera distance and the contour size is directly dependent on each other. The larger the contours, the larger the camera distance should be. The small contour of $0.1 \mathrm{~mm}$ represents a stable point for this task. Further experiments have been done with $500 \mathrm{~mm}$ camera distance. Another experiment has shown irregular reflections on the tooling side, which leads to wrong detection.

After determining the influences in the laser point detection, another experiment was carried out. Here, the laser spot was only projected on the non-tool side (which also corresponds to reality) at a camera distance of $500 \mathrm{~mm}$. Thus, sources of error, such as the detection of large contours and random reflection of the smooth tool side, can be avoided. The evaluated results of the experiments are shown in $(4 a, 4 b)$. Both figures show a pixel value difference within the plate on the $\mathrm{X}$-axis. This was most likely due to the random reflection of the tool side. The pixel value deviation on the $\mathrm{Y}$ axis between two plates has arisen due to the component thickness difference.

Figure $4 \mathrm{c}$ shows, that no difference between two projectors can be seen in the correlation value, even though the contours were projected once with a projector and once with two projectors onto a $5 \mathrm{~mm}$ and a $2 \mathrm{~mm}$ thick component. The correlation value was between $70 \%$ and $80 \%$. Thus, it could be stated that the number of projectors has no significant influence. The component reflection plays a certain role in laser point detection. However, this can be avoided by the increasing camera distance to the component and the reducing projection contour (for example $0.1 \mathrm{~mm}$ ). Furthermore, different filters to reduce noise and algorithm for subpixel detection have been implemented. Later on for $3 \mathrm{D}$ reconstruction, new software version has been used to detect corresponding $2 \mathrm{D}$ laser points on the image. 

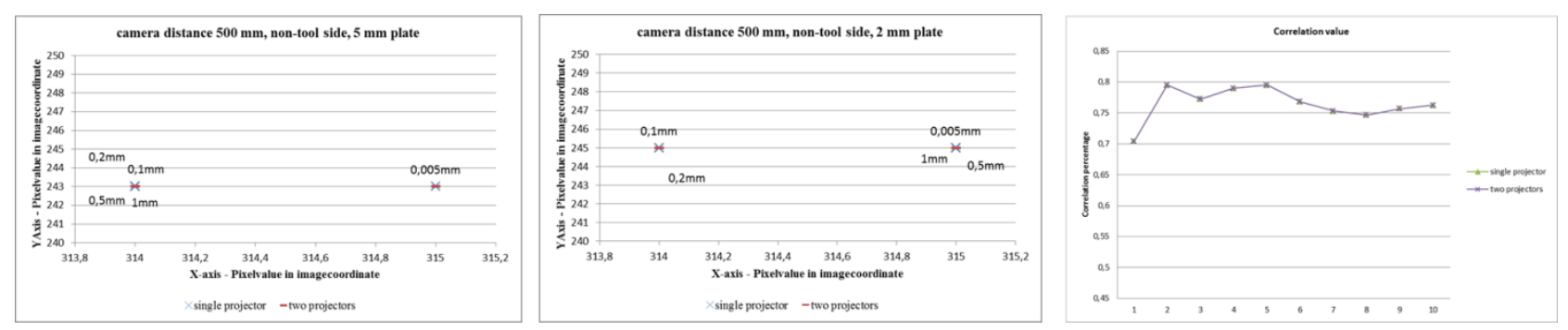

Figure 4: (4a) Experiment on $5 \mathrm{~mm}$ plate; (4b) experiment on $2 \mathrm{~mm}$ plate; (4c) achieved correlation value of detected laser point; for all experiments camera distance was $500 \mathrm{~mm}$ with different contours by using one and two laser projectors respectively

\section{Geometric and Hand-Eye camera calibration}

DLR Augsburg is pursuing research since few years on geometric calibration method for intrinsic parameters and HandEye-Camera calibration method for extrinsic parameters related to thermography camera. Concepts and previous experiment results have been published in conference papers [3, 13 and 14]. In paper [4] a detail analysis about geometric thermography camera calibration for both objectives (wide-angle lens with $12 \mathrm{~mm}$ focal length and normal-lens with $27 \mathrm{~mm}$ focal length) have been presented and discussed. Hand-eye calibration method was developed and validated with normal-angle with $27 \mathrm{~mm}$ focal length lenses [14]. To generate 3D thermography for both methods (from known 2D-3D correspondences method as well from kinematic coordinate chain method), the intrinsic and extrinsic parameters of a thermography camera should be obtained prior to mapping of thermal images to the 3D surface of an object. These parameters have not been determined in previous experiment for new experimental setup (i.e. $500 \mathrm{~mm}$ camera distance with $27 \mathrm{~mm}$ focal length and different angle of mounted camera) for 3D thermography. These parameters have been generated now by using Matlab calibration toolbox and DLR developed Hand-Eye-toolbox. New experiment results have been verified with previous results [14] and shows similarity up to few pixel differences. The difference comes due to the use of difference calibration pattern size and preparation. Since the experiment was focussed on implementation of texture mapping method, the calibration pattern was not prepared highest accuracy. This known inaccuracy, which will effect later on in 3D-thermography model, was not considered in this paper. Figure 5 represents the (a) calibration images, (b) camera view positions and (c) experiment setup. These experiments have been performed with up to 4 iteration and no significant changes have been observed after 3 iteration processes. Results of intrinsic as well as of extrinsic parameters are presented in table 1 and 2 . The re-projection error of \pm 0.15 (see figure 6 ) $\mathrm{mm}$ has been achieved with a grid size of $20 \mathrm{~mm}$ and 10 thermography images.

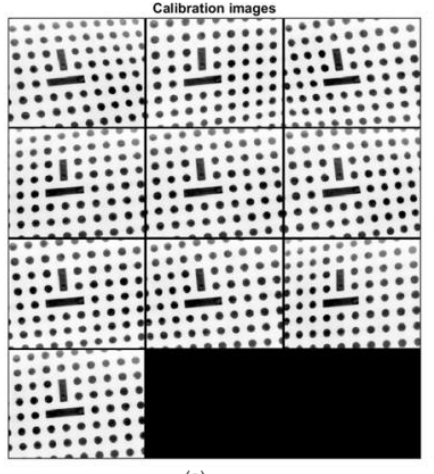

(a)

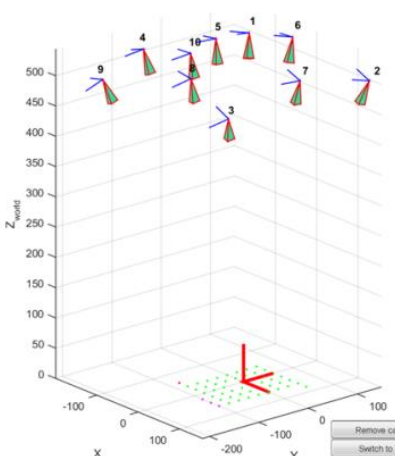

(b)

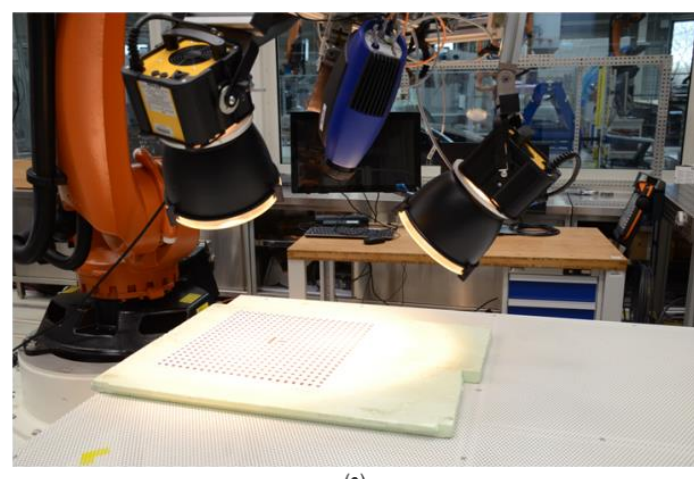

(c)

Figure 5: (a) calibration images, (b) camera view positions and (c) experiment setup

Table 1: intrinsic calibration parameters after 4th iteration

\begin{tabular}{|c|c|}
\hline Focal length & {$[1972,25761967,28746] \pm[5,6907 \quad 5,64439]$} \\
\hline Principal point & {$[311,06954250,61192] \pm[5,148644,29913]$} \\
\hline Skew & {$\left[\begin{array}{lllll}0 & 0 & 90 & 0\end{array}\right]$} \\
\hline Distortion & {$\left[\begin{array}{llllll}-0,25504 & -3,90877 & 0,00015 & -0,00021 & 0\end{array} \pm\left[\begin{array}{lllll}0,04094 & 1,34223 & 0,00044 & 0,00036 & 0\end{array}\right]\right.$} \\
\hline Pixel Error & {$[0,076190,07325]$} \\
\hline
\end{tabular}


Table 2: extrinsic Hand-Eye calibration results for $500 \mathrm{~mm}$ camera distance

\begin{tabular}{|c|c|c|c|}
\hline \multicolumn{2}{|c|}{ Rotation in $^{\circ}$} & \multicolumn{2}{c|}{ Translation in $\mathrm{mm}^{\circ}$} \\
\hline A & 150,581 & $\mathrm{X}$ & 17,711 \\
\hline B & 0,1963 & $\mathrm{Y}$ & 38,2402 \\
\hline C & 0,1708 & $\mathrm{Z}$ & 705,076 \\
\hline
\end{tabular}

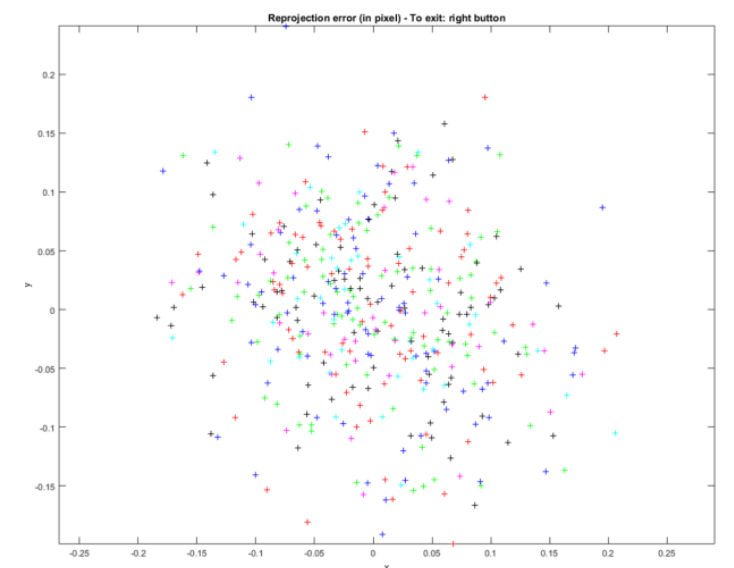

Figure 6: Re-projection error after Hand-Eye calibration

\section{3D Visualisation}

Thermography measurement and evaluation of each component constitutes a different case study, given not only the different existing geometries and compositions, but also their manufacturing process. Finding measurement parameters for different components are iterative (experimental or simulative) process. Inspection of complex component requires geometric information for the evaluation, heat propagation quantification and precise defects location in space. Therefore, 3D evaluation by means of 3D visualisation of measurement results gives complex but complete 3D defect propagation knowledge. In following sections the applied 3D thermography visualisation approaches are explained in detail.

\subsection{D-3D Point correspondence based texture mapping}

In order to establish 2D-3D correspondence relationship, most of the approaches use either geometric features of the object or previously applied marker points on the object. The projected features of new concept do not have the properties of common markers. Markers, physically or optically to the component, are given in terms of size, shape and distance to each other, whereas the used laser point is not. This concept follows the approach of using laser pointer as contactless marker point. The developed automated 3D thermography process has been demonstrated on complex geometry along with process chain. Figure 7a represents experimental setup. To achieve higher accuracy (see details in section 2) 3D real surface geometry has been generated in CATIA from measured point cloud by using highly accurate device T-scan. After measuring the real geometry, the corresponding 3D points were constructed in CATIA. There are nine such points per measurement field. The first three measurement fields are highlighted in color (see figure $7 \mathrm{~b}$ ). The defined nine correspondent points per measurement field are projected onto the component by the laser projector. Prior to that, the component is calibrated with known reflector's position to the laser projector. Those constructed corresponding points are used to generate offline robot program. Thus, the robot moves with the thermography end effector to the respective measurement field and the thermal camera captures the projected laser points. The image recorded by the measurement position was then evaluated in Matlab (see also section 2) and displayed together with the nine reference points in image coordinate. Hence, each image receives the reference points for the respective position. To be able to reconstruct in $3 \mathrm{D}$, the projection matrix, which operates in homogenous coordinate system, has to be calculated from 2D-3D data set. This data set has to be transformed from Cartesian coordinate in homogenous coordinate by including additional component $w$. 3D point with coordinates $\{x, y, z\}$ and a point with homogeneous coordinates $\{x, y, z, w\}$ are equivalent as long as $w=1$. The same applies for inhomogeneous $2 D$ points, they also get a third coordinate $w$. Due to fourth dimension to world point (1 row with four columns) the transformation matrix or image projection matrix $M$ has $4 \times 4$ dimensions. This additional fourth dimension (last row of the Projection matrix) represents perspective and scaling factor. The scale factor will almost always be 1 and the perspective part will be all zeros except when modelling cameras. Since $\mathrm{M}$ relates to homogenous data sets, scale and perspective factor can be neglected. Therefore, projection matrix has the dimension of $3 \times 4$ and contains intrinsic and extrinsic information. This matrix can be calculated by using mathematical camera model of Tsai, Zhang and DLT, which is built on the principles of the pinhole camera. It models the central projection of $3 D$ points through the center of projection onto the image plane. The generated intrinsic parameters $K \in \mathbb{R}^{3 \times 3}$ and extrinsic parameters $[R \mid t] \in \mathbb{R}^{3 \times 4}$ represents altogether corresponding mapping in homogeneous coordinate, $3 D$ point $P \in \mathbb{R}^{4}$ to $2 D p \in \mathbb{R}^{3}$ image point, which is defined by $\mathbf{p}=\boldsymbol{K}[\mathbf{R} \mid \mathbf{t}] \mathbf{P}$. In 
general, non-linear approaches are very robust to image noise and can calculate extrinsic parameters with less corresponding points than classical DLT. But non-linear approaches like PnP, LHM, POSIT etc. require a good initial guess for pose estimation. Additionally linear solution like DLT can be used as initial guess in future for non-linear solution. Intrinsic camera parameters from geometric camera calibration have been used not only for laser point detection but also DLT algorithm. However, due to less noisy image projection, matrices are calculated per measurement field by using a mathematical approach (DLT - Discrete Linear Transformation and SVD Single Value Decomposition). The projection matrix contains the relationship between the $2 \mathrm{D}$ image and the $3 \mathrm{D}$ part because the reference points are related to the part coordinate system.

These generated projection matrix per measurement field are then taken to calculate an image position for each model vertex. All images are taken with overlap at their boundary areas. To stich images with each other without overlap by using graphic card, one single texture is generated from all images in a post-processing step. The images are merged together in the most quadratic way possible. Software inputs required for texture mapping are 3D Model vertices, 2D undistorted thermography image folder, correspondence data set and intrinsic parameters. After loading the information to graphic card, it takes few minutes for 3D texture mapping. In this case for 12 images it took less than one minute. This texture mapping and post processing method is described in detail in the master thesis of Pascal Keller "A new application for texture mapping using 2D-3D Correspondences". In Addition to 3D visualisation, 3D defect localisation method has been implemented. By clicking on defect location in the software application, 3D coordinates in components coordinate are displayed. This information can be provided to laser projector to show the desired defect location on components surface. Further development of 3D localisation method is presented in section 5 . Figure 8 represents the automated 3D thermography visualisation and defect localisation process. The automation process starts with geometric camera calibration and the following steps are shown with red arrows.

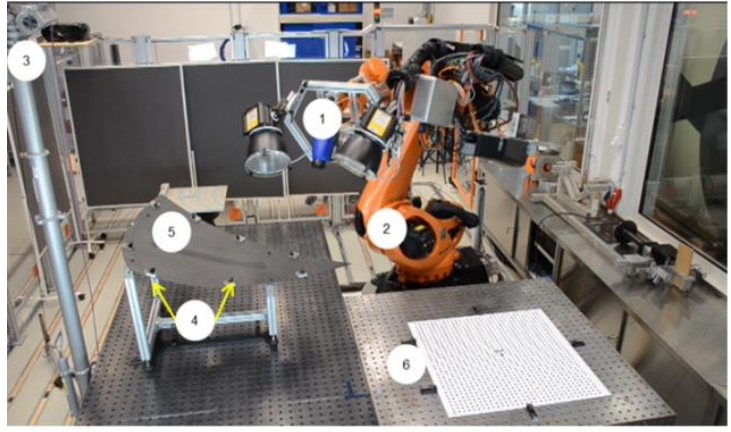

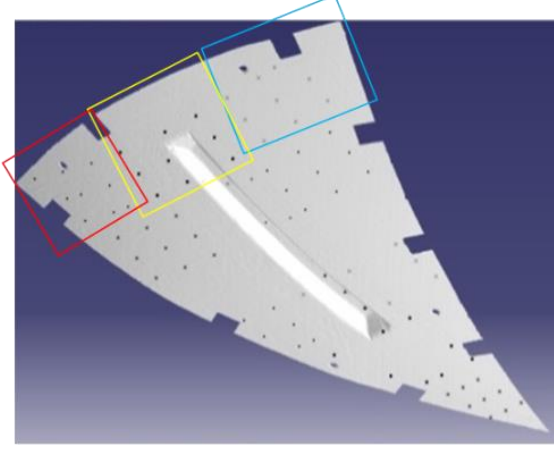

(b)

Figure 7: (a) Experimental setup (b) highlighted measurement field; Test setup consist of mainly by camera (1), robot (2), laser projector (3), reflectors for laser projector to component calibration (4), component (5), calibration pattern (6)

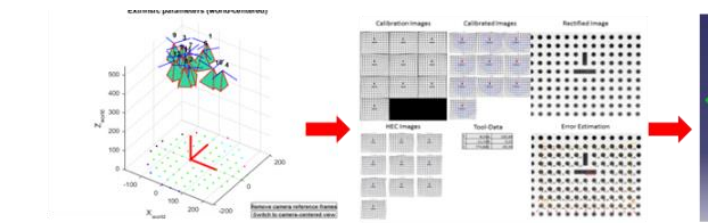

Geometric camera calibration

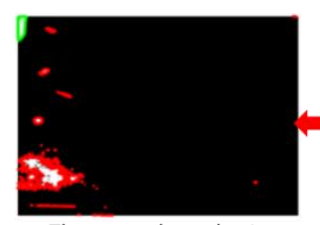

Thermography evaluation

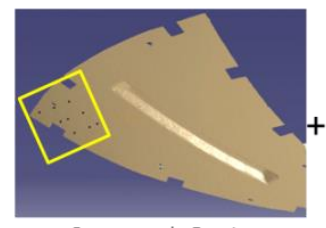

Constructed 3 D points
Hand-Eye calibration

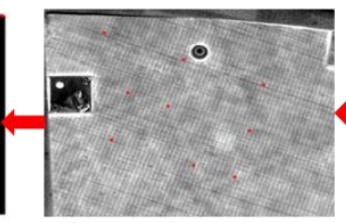

Thermography measurement

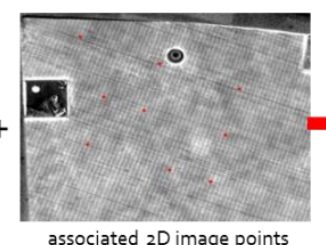

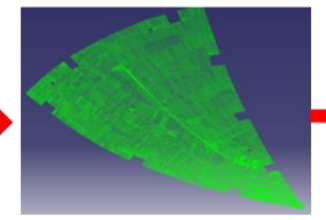

$3 \mathrm{D}$ geometry measurement

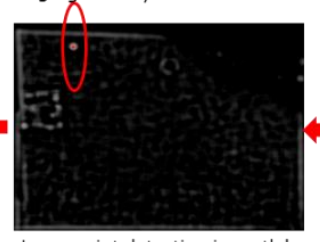

Laser point detection in matlab

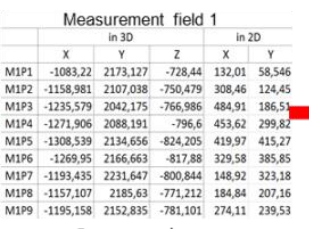

Correspondence

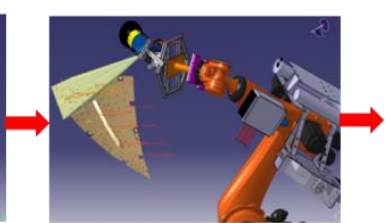

Model setup for offline program

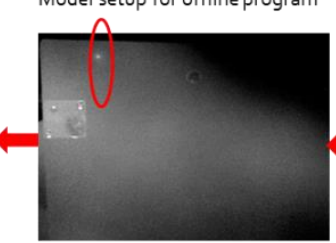

Thermography live image

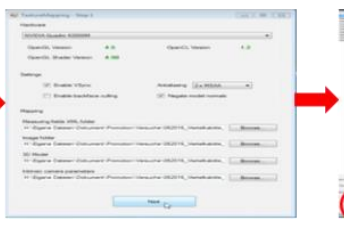

Texture Mapping

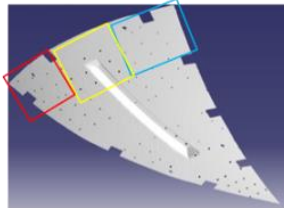

Measurement field setting
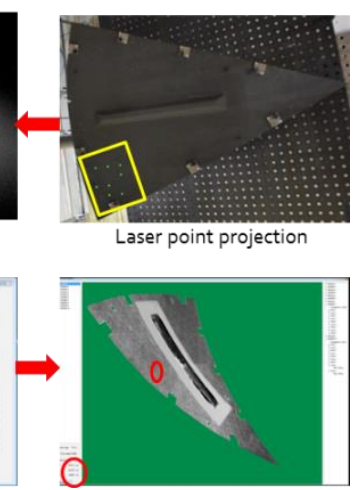

$3 \mathrm{D}$ defect localisation

Figure 8: automated 3D thermography visualisation and defect localisation process

A further experiment has been done to demonstrate the complete process chain on large component. For that a fuselage with the dimensions of $4 \mathrm{~m}$ in diameter and $2 \mathrm{~m}$ width are taken for the experiment. In order to get high 3D mapping 
accuracy, the real surface of the fuselage has been measured and total number of 1640555 vertices generated. Due to robots reachability, measurement distances for thermography were between 1 and $1.2 \mathrm{~m}$. Pixel resolution for approx. 1.2 $\mathrm{m}$ camera distance was 0.6 Pixel / $\mathrm{mm}$. Due to manufacturing and engineering edge, $50 \mathrm{~mm}$ from border side has not been measured. In total 105 measurement fields were required to cover the complete surface. Figure 9a represents the vertices and real surface. The constructed corresponding 3D points in CATIA are enlarged and illustrated in 9b. Offline Robot program was generated after measuring component's base position (see figure 10a). Hereby, tool centre point has been set from previous Hand-Eye calibration results. Due to large surface area two laser projectors were calibrated for each half of the surface area. For each measurement field 9 laser points have been projected and captured by the camera. After that thermography measurement has been executed with $0.02 \mathrm{~Hz}$ frequency. To avoid lateral thermal diffusion during thermography measurement execution, measurement field has been chosen arbitrary. Total measurement time was with $0,02 \mathrm{~Hz}(\mathrm{EP}=1 ; \mathrm{MP}=3): 200(\mathrm{~s}) \times 105=5.83 \mathrm{hrs}$, which is compared to conventional water coupled ultrasonic method very less. Figure 10b represents experiment setup. With the new developed software it took less than 2 minutes to visualise 3D thermography (see figure 10c). By a right click on the surface area (see figure 10c) $3 \mathrm{D}$ coordinates can be displayed to project or analyse critical position.

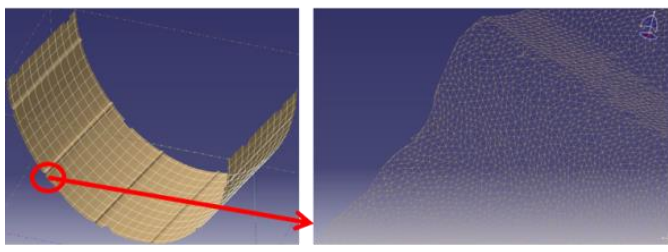

(a) 3D surface generation

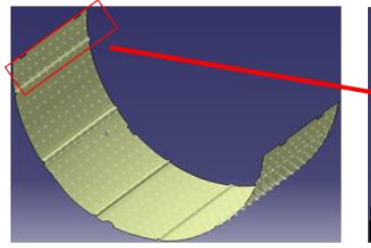

(b) Measurment field setting

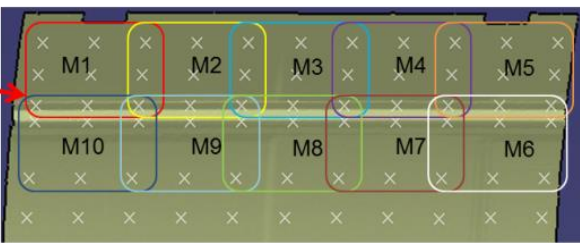

Figure 9: (a) 3D surface generation and vertices; (b) Measurement field setting

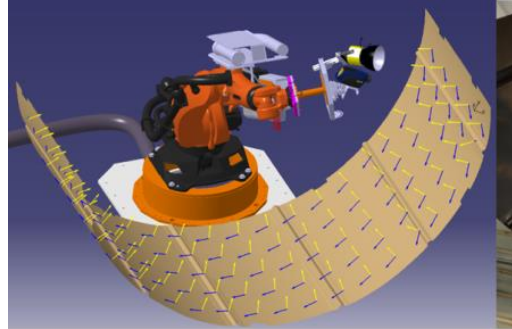

(a) Generated Offline-Roboterprogram

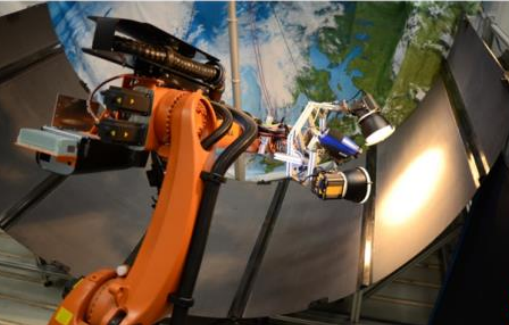

(b) Thermography at fuselage

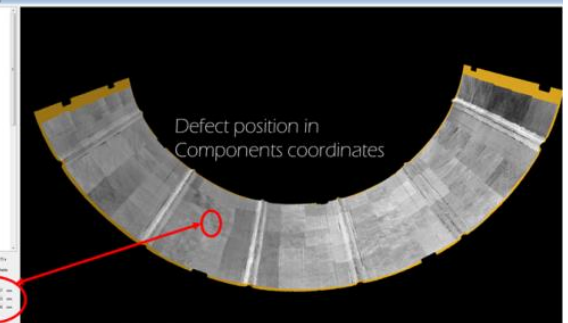

(c)3D visualisation and defect localisation

Figure 10: (a) generated offline-Program; (b) Experimental setup; (c) 3D visualisation and defect detection

\subsubsection{Texture mapping accuracy}

After texturing, a rough error study was performed. Figure 11 shows two surface areas (arbitrarily chosen) for accuracy testing. Two images were stitched at the red dashed line. The individual rovings are about 1 to $2 \mathrm{~mm}$ wide. The figure clearly shows that the rovings are more offset at the edge area than at the centre of the picture. A detailed examination of the method is planned for the next experiment. However, it can be assumed that the larger error component is caused by the camera calibration parameters. Due to the camera distance to the object, the error at the edge area is greater than in the centre of the image. Certainly, there are also errors in laser projection, point recognition, area reconstruction from the point cloud, the texture mapping, etc. In the further series of experiments, these topics will be focused.
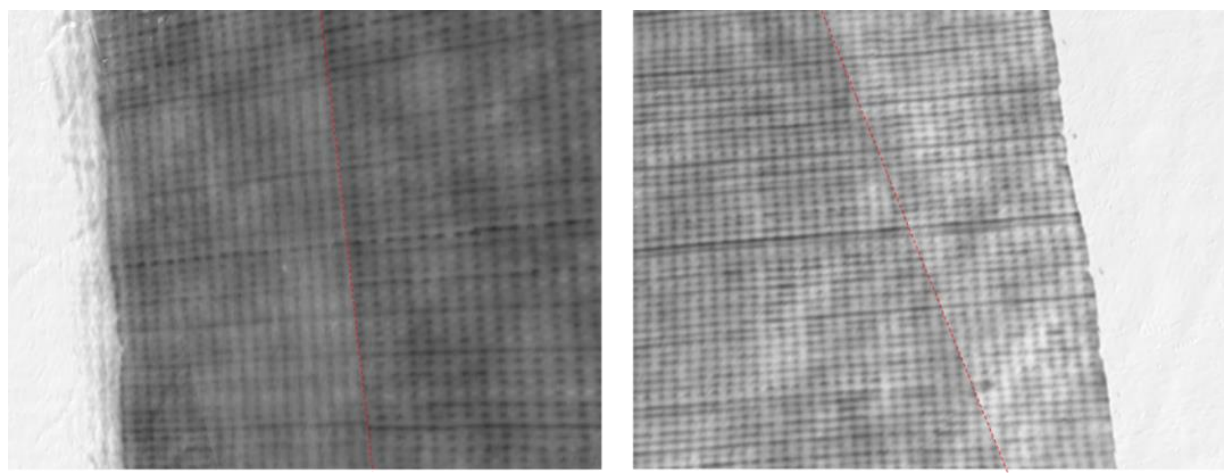

Figure 11: Texture mapping accuracy 


\subsection{Robot based 3D texture mapping}

While 3D texture mapping, using robot kinematic coordinates relationship, camera pose has to be calculated related to component. In previous sections and papers, robot's drawbacks for automated 3D thermography have been analysed. There are many different approaches for measurement of six dimensional camera pose of an industrial robot, like robot guidance for machine vision system or robot based texture mapping or camera calibration [15, 16 and 17]. All these approaches are providing possible way to calculate camera pose in order to improve robot deficiency. The proposed approach in this paper has been illustrated in figure 2. The arrows represent the $4 \times 4$ transformation matrix from one coordinate to next coordinate. The homogeneous transformation matrix (see previous section) is a $4 \times 4$ matrix that is defined for mapping a position vector from one coordinate system to another. Last row of transformations matrix represents scale and perspective coefficients, which can be neglected. Therefore, transformation matrix provides at the end $3 \times 4$ projections matrix. In order to implement the algorithm (based on kinematic coordinate relationship) in the new software, $3 \times 4$ projection matrix is required. This projection matrix contains the extrinsic parameters in terms of camera pose related to robot and component.

A robot manipulator is an electronically controlled mechanism and composed of an assembly of links and joints. Links are defined as the rigid sections that make up the mechanism and joints are defined as the connection between two links. The attached thermography end-effector at flange is rigid joint. Therefore transformation matrix $\mathrm{FT}_{\mathrm{C}}$ (see figure 2) between flange and camera plane needs only to be calculated once. These calibrations have been performed in section 3. Without a pre-calibration (hand-eye calibration) of camera, the six dimensional pose measurements for every new image is not possible. In parallel geometric camera calibration is also required in order to undistort the images before it is mapped to component. The $\mathrm{RT}_{\mathrm{B}}$ transformations matrix between robot and component needs to calculate whenever the component's positions change. In general, the world coordinate does not change and the relation between robroot and world coordinate are calibrated through KUKA robot language. This $W T_{R}$ transformations matrix can be read from machine.dat file. Furthermore, KUKA software delivers the entire transformation matrix $\mathrm{RT}_{\mathrm{F}}$ between robroot and flange for all the new positions. $\mathrm{RT}_{\mathrm{B}}$ and $\mathrm{W} \mathrm{T}_{\mathrm{B}}$ transformation matrix are generated through measuring the component's position by using 4 point method, provided by KUKA. The $\mathrm{BT}_{\mathrm{C}}$ transformation matrix can be calculated by multiplying the other matrices. $B T_{C}=\left(R T_{B}\right)^{-1} \cdot R T_{F} F T_{C}$. This has to be done for every measuring field.

To verify the system, a new experimental setup has been prepared (see figure 12). The component was $1000 \mathrm{~mm}$ long and $500 \mathrm{~mm}$ wide. With a measurement distance of $500 \mathrm{~mm} 30$ measurement fields were required to cover the complete surface. After doing the hand-eye calibration and geometric calibration the component's base was measured by using 4 point method, provided by KUKA. Offline robot program has been generated using measured TCP and base position. Robot program was generated in base coordinate system. After that, the component was measured with thermography camera by using $0.4 \mathrm{~Hz}, 0.1 \mathrm{~Hz}$ and $0.05 \mathrm{~Hz}$ parameters. 30 images per frequency, intrinsic parameters, dat file of robot program, which contains all measured robot positions, and rigid transformation matrices are provided to software to calculate the projection matrix. The results have been illustrated in figure 13.

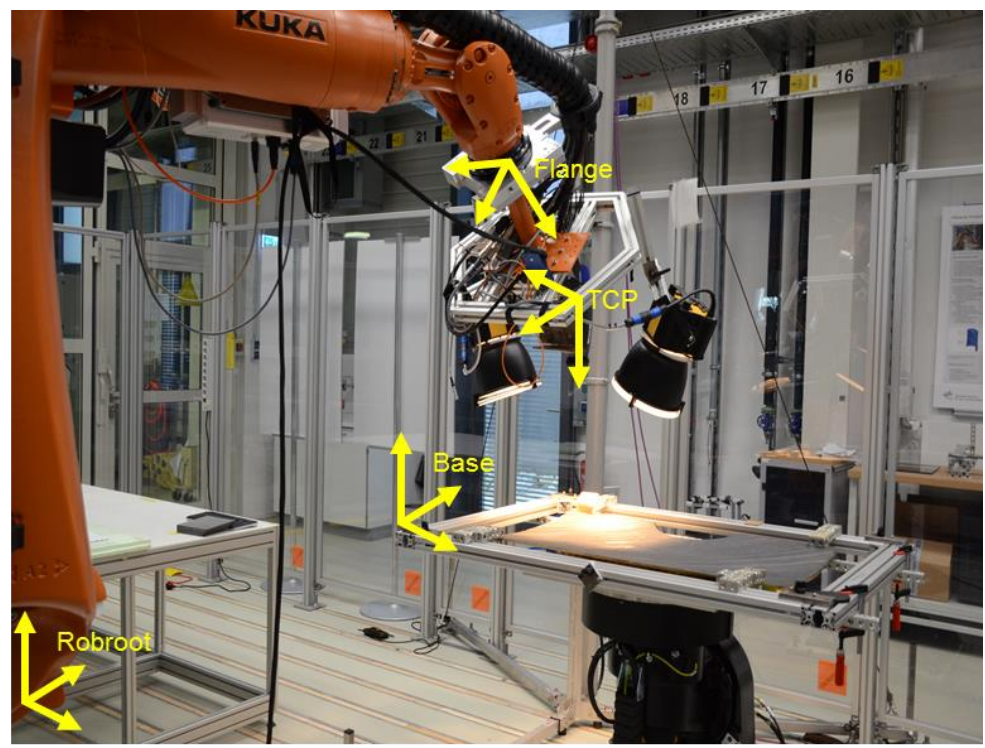

Figure 12: experimental setup for kinematic relationship based algorithm

\section{3D Thermography in CATIA}

As discussed in introduction, examiner has to evaluate every thermography image one by one, which is unhandy and less intuitive method for large number of numerical data. A possible solution has been developed to automate thermography evaluation process. The excitation frequency in lock-in thermography is chosen by its dependence on the sample's thermal characteristics and it's geometrical dimensions. The lower the frequency of the thermal waves are, the slower the velocity in the test specimen and deeper its penetration into the test specimen. Thus, for the measurement 0.4 $\mathrm{Hz}, 0.1 \mathrm{~Hz}$ and $0.05 \mathrm{~Hz}$ frequency has been chosen, the penetration depth can be calculated using $\mu=\sqrt{ }\left(\alpha / \pi^{*} \mathrm{f} H z\right)$ 
equation, where a represents thermal conductivity of carbon fibre composite. In general, thermal conductivity has to be determined experimentally. The penetration depths for the chosen frequencies (with $0.004 \mathrm{~cm}^{2} / \mathrm{s}$ thermal conductivity) are $0.56 \mathrm{~mm}, 1.2 \mathrm{~mm}$ and $1.6 \mathrm{~mm}$. The real surface was measured with Tscan and a surface with vertices has been generated in CATIA (see section 4 more details). Furthermore, for the three thermal depth three offset surfaces with appropriate parameters $(0.56 \mathrm{~mm}, 1.2 \mathrm{~mm}$ and $1.6 \mathrm{~mm}$ ) have been generated (see figure 13f) and exported as stl file, so that the thermography results can be visualised in accordance to their depth.

Thermography images are then mapped with their belonging offset surfaces in the software and exported as VRML file format. This file contains all information of image colours associated with 3D vertices with their corresponding orientation and positions. At the next step the VRML file together with a merged image will be imported again in CATIA. This allows to illustrate all thermography images together corresponding to their depth. Figure 13a shows the plies and implanted kapton foil in different depth. Figure 13b, 13c and 13d illustrates the 3D thermography results in three different depths. In these figures, it can be recognised, that with increasing thermal depth, more kapton foil and real manufacturing defects are getting visible. Figure 13 e represents the 3D thermo-tomography model. This 3D thermo-tomography model can now easily be analysed by clicking the CATIA's show option to visualise the results of desired thermal depth. In this way other manufacturing tolerances and coherent influences (for example ultrasonic welding during preform process) can be evaluated. Furthermore, structural analysis can be compared with thermography results as both 3D thermos-tomography and structural analysis are using the same software CATIA. This thermo-tomography model represents a simplified version of real computer tomography. Evaluation of 3D damage propagation is now easier. The implanted kapton foil in different depth has been detected with the chosen measuring frequency. After mapping all the 90 thermography images, the 3D location and size of those kapton foils have been exported from CATIA to laser projector to project directly on components surface. This allows to avoid marking the damage area and robot or employee can take required step. For an example, only desired damages, which need to be repaired, can be projected. In figure 14a few of damages are marked with yellow line. As results, only these damages (dimension and location) are exported and projected (see figure 14c).

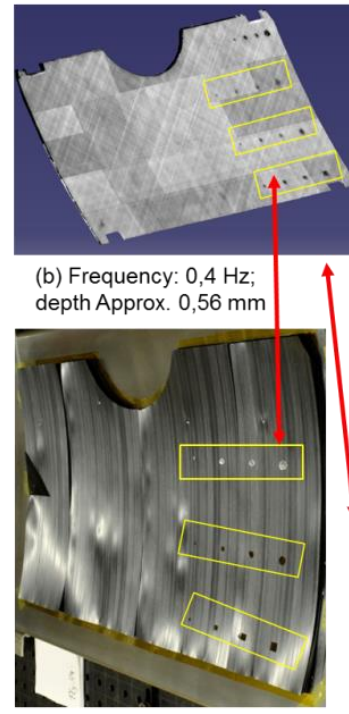

(a) Capton foil as artificial defect

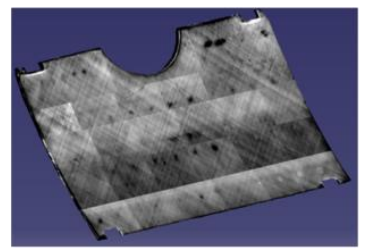

(c) Frequency: $0,1 \mathrm{~Hz}$; depth Approx. $1.2 \mathrm{~mm}$

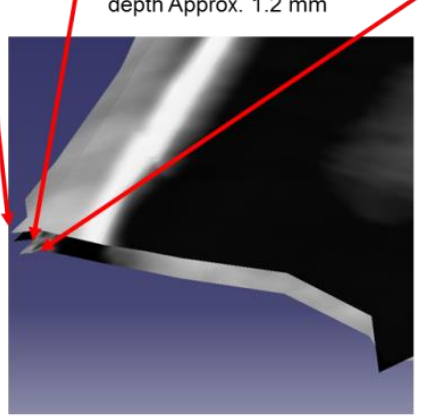

(e) 3D-Thermotomography

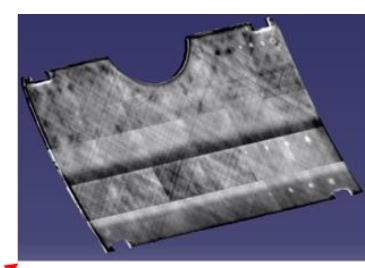

(d) Frequency: $0,05 \mathrm{~Hz}$ depth Approx. $1.6 \mathrm{~mm}$

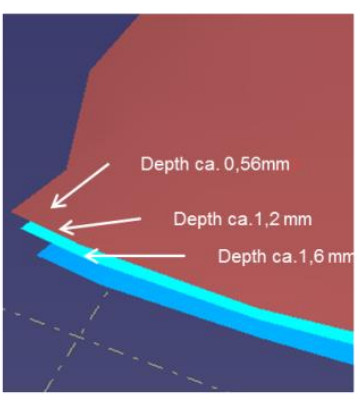

(f) Vercices according to measurement depth

Figure 13 (a) Preforming process; (b) 3D thermography results in $0.56 \mathrm{~mm}$ depth; (c) 3D thermography results in $1.2 \mathrm{~mm}$ depth; (c) 3D thermography results in $1.6 \mathrm{~mm}$ depth; (d) 3D-Thermo-tomography model; (e) vertices with different offset

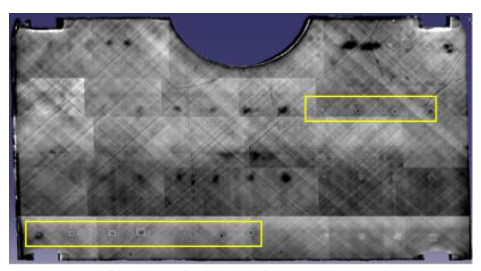

(a) Marking of critical defect position and size

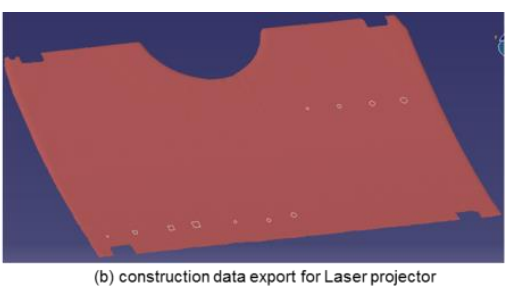

(b) construction data export for Laser projector

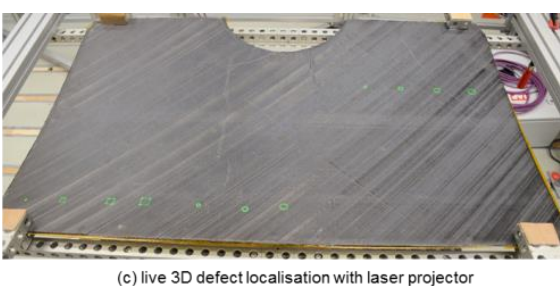

c) live 3D defect localisation with laser projector

Figure 14: (a) Critical defects; (b) exporting defect location and dimensions from catia; (c) projection of defects with their dimensions 


\section{Conclusions}

In this series of experiments, thermographic images were 3D visualized using the 2D-3D point correspondence method and robot based kinematic relationship chain method. At first, to minimize the drawbacks of robot based automated inline thermography, concept of 2D-3D corresponding method is described. Secondly feasibility of this concept is experimentally verified. In this experiment, it could be shown that the projected contour size and the camera distance to the component are dependent to each other, while detecting the laser point. For a reliable laser point detection smaller circles with $0.1 \mathrm{~mm}$ diameter and the largest possible camera distance is recommended. The correlation value in this test series was between $70 \%$ and $80 \%$. Afterwards, DLT method has been applied to calculate projection matrix out of 2D-3D corresponding data set. Using two experimental setups, one on large single curved component (fuselage) and second on a double curved component, said method has been validated. The results confirm the feasibility of the new concept. An automated thermography process chain and visualisation of the results in 3D were presented. The individual process steps of the automated inline thermography were reported as well. After that, a system accuracy analysis overview was carried out and the possible source of error is shown. An exact overall system analysis and optimization of the method is planned for the next experiment. This 2D-3D corresponding method can also be applied without any manipulator like robot. Only laser projector and component should be calibrated to each other. This simplification allows this system to applicable in laboratory. Furthermore, robot based 3D visualisation method has been implemented. Results have been validated through experiment. System accuracy analyses of both methods on same component in same experimental setup are to be done in next experiment. Besides that a new approach for 3D evaluation by means of 3D-thermotomography has been showed. This new approach helps examiner to analyse coherent influences of manufacturing process over the surface and in their depth. Another future goal is to implement alternative non-linear 2D-3D corresponding method, like PnP, POSIT etc.

\section{REFERENCES}

[1] Volker Carl, Konstantin Eisler; „3D-Formerfassung im Infraroten und Sichtbaren sowie Techniken zum Visualisieren“; Thermografie-Kolloquium 2011

[2] Thomas Schmidt, Somen Dutta; "Automation in production integrated NDT using thermography". NDT in Aerospace 2012

[3] Somen Dutta, Thomas Schmidt; "New concept for higher Robot position accuracy during thermography measurement to be implemented with the existing prototype automated thermography end-effector utilising an industrial robot and laser system". QIRT 2014.

[4] Thomas Schmidt, Christoph Frommel; "Geometric Calibration for thermography cameras", 7th International Symposium on NDT in Aerospace, 2015

[5] Stephen Vidas, Peyman Moghadam; "HeatWave: A handheld 3D thermography system for energy auditing"; Energy and Buildings. Science Direct, Elsevier

[6] Renkielska A., Nowakowski A., Kaczmarek M., et al., Burn "depths evaluation based on active dynamic IR thermal imaging - a preliminary study". Burns; 32: pp. 867-875, 2006.

[7] Y. Horry, K. Anjyo, and K. Arai. "Tour into the picture: using a spidery mesh interface to make animation from single image"; In Proc. SIGGRAPH, pages 225-232, 1997.

[8] A. Criminisi, I. Reid, and A. Zisserman. Single view metrology. IJCV, 40(2):123-148, 2000.

[9] P. F. Strum and S. J. Maybank, "A method for ineractive 3d reconstruction of piecewise planar objects from single views"; In Proc. BMVC, pages 265-274, 1999.

[10] P. E. Debevec, C. J. Taylor, and J. Malik "Modeling and rendering architecture from photographs: a hybrid geometry and image based approach"; In Proc. SIGGRAPH, 1996.

[11] M. Wilczkowiak, E. Boyer, and P. Strum. Camera calibration and 3d reconstruction from single images using parallelepipeds. In In Proc. ICCV, pages 142-148, 2001.

[12] Bryson R. Payne, James F. Lay, Markus A. Hitz; "Automatic 3D Object Reconstruction from a Single Image"; ACM SE '14, Mar 28-29 2014, Kennesaw, GA, USA

[13] Thomas Schmidt, Somen Dutta "CFRP manufacturing process chain observation by means of automated thermography," 5th International Symposium on NDT in Aerospace, Singapore, 2013.

[14] Thomas Schmidt, "Hand-eye-calibration of a robotized thermography end-effector", 6th Internationa Symposium on NDT in Aerospace, 12-14th November 2014, Madrid, Spain

[15] R. A. Boby, S. K. Saha ; "Single Image based Camera Calibration and Pose Estimation of the End-effector of a Robot”; 2016 IEEE International Conference on Robotics and Automation (ICRA);Stockholm, Sweden, May 1621, 2016

[16] Luis Pérez, Íñigo Rodríguez ,†, Nuria Rodríguez,†, Rubén Usamentiaga and Daniel F. García; "Robot Guidance Using Machine Vision Techniques in Industrial Environments": A Comparative Review; 5 March 2016; sensors

[17] Marco Ulrich, Andreas Forstner and Gunther Reinhart; "High-Accuracy 3D image stiching for robot based inspection systems"; Image processing ICIP, IEEE 